\title{
Nanomaterial-based receptor conjugates for capture and rapid detection of Salmonella Enteritidis
}

\author{
Kiara Cousin ${ }^{1}$, Boniface Tiimob ${ }^{2}$, David Baah ${ }^{3}$, Cesar Fermin ${ }^{1}$, Temesgen Samuel ${ }^{1}$, Woubit Abdela ${ }^{1}$
}

1. Department of Pathobiology, Tuskegee University, Tuskegee, AL 36088, United States.

2. Department of Material Science and Engineering, Tuskegee University, Tuskegee, AL 36088, United States.

3. Department of Chemical Engineering, Tuskegee University, Tuskegee, AL 36088, United States.

According to the Centers for Disease Control and Prevention (CDC), an estimated 1 in 6 Americans become ill by consuming foods or beverages contaminated by disease causing pathogens each year [1]. Foodborne diseases have become a significant public health concern throughout the globe with an increase in incidence over the last two decades [2]. Salmonellosis is one of the most important bacterial diseases of food safety concern; and is caused primarily by Salmonella species such as Salmonella Enteritidis and Salmonella Typhimurium [3]. The World Health Organization (WHO) has shown statistics of tens of millions of new human cases and more than 100,000 deaths every year from individuals with symptoms that include fever, abdominal pain, diarrhea, nausea, and vomiting $[3,4,5,6]$.

Conventional methods using selective media for culture and colony counting as well as immunological based identification methods and polymerase chain reaction (PCR) have been deemed laborious, time consuming, or may require pretreatment of samples [7]. Therefore, there is the need for better, rapid, sensitive, and economical identification and detection techniques for foodborne pathogens. Nanomaterial-based biosensors have been used as means of prompt identification. This study aims to identify potential recognition receptors or elements for Salmonella detection using gold (Au) nanoparticles, magnetic nanoparticles (AuNP, MNP), and biomolecules to design nano-conjugates for pathogen capture.

Construction of antibody functionalized MNP (AFMNP) was achieved by optimizing techniques derived from Zhang, Carr, and Alocilja, (2008) [8] and Wang et al., (2014). Upon purification of DNA probes, functionalized magnetic nanoparticles (fMNP) were coupled with a universal anti-Salmonella antibody (Abcam ab8273) via a disulfide cleaved dithobis [succinimidylpropionate] (DSP) conjugate to form an AFMNP sandwich structure. Pathogen capture of Salmonella Enteritidis was achieved by combination of sandwich structure with bacteria and subsequent separation via magnet. Samples were analyzed using CytoViva ${ }^{\circledR}$ Hyperspectral Imaging system and transmission electron microscopy.

Preliminary study on this AFMNP showed promise in capturing Salmonella Enteritidis bacteria in situ. Successful conjugation of the MNP with antibody was determined with transmission electron microscopy and hyperspectral image analysis while pathogen-capturing effect of the AFMNP was determined by inoculating captured pathogens on the AFMNP Brilliant Green Sulfa agar plates. The results showed that the AFMNP was successful in capturing Salmonella Enteritidis in solution. Further studies are being implemented using food substrates to evaluate the effectiveness of AFMNP. 

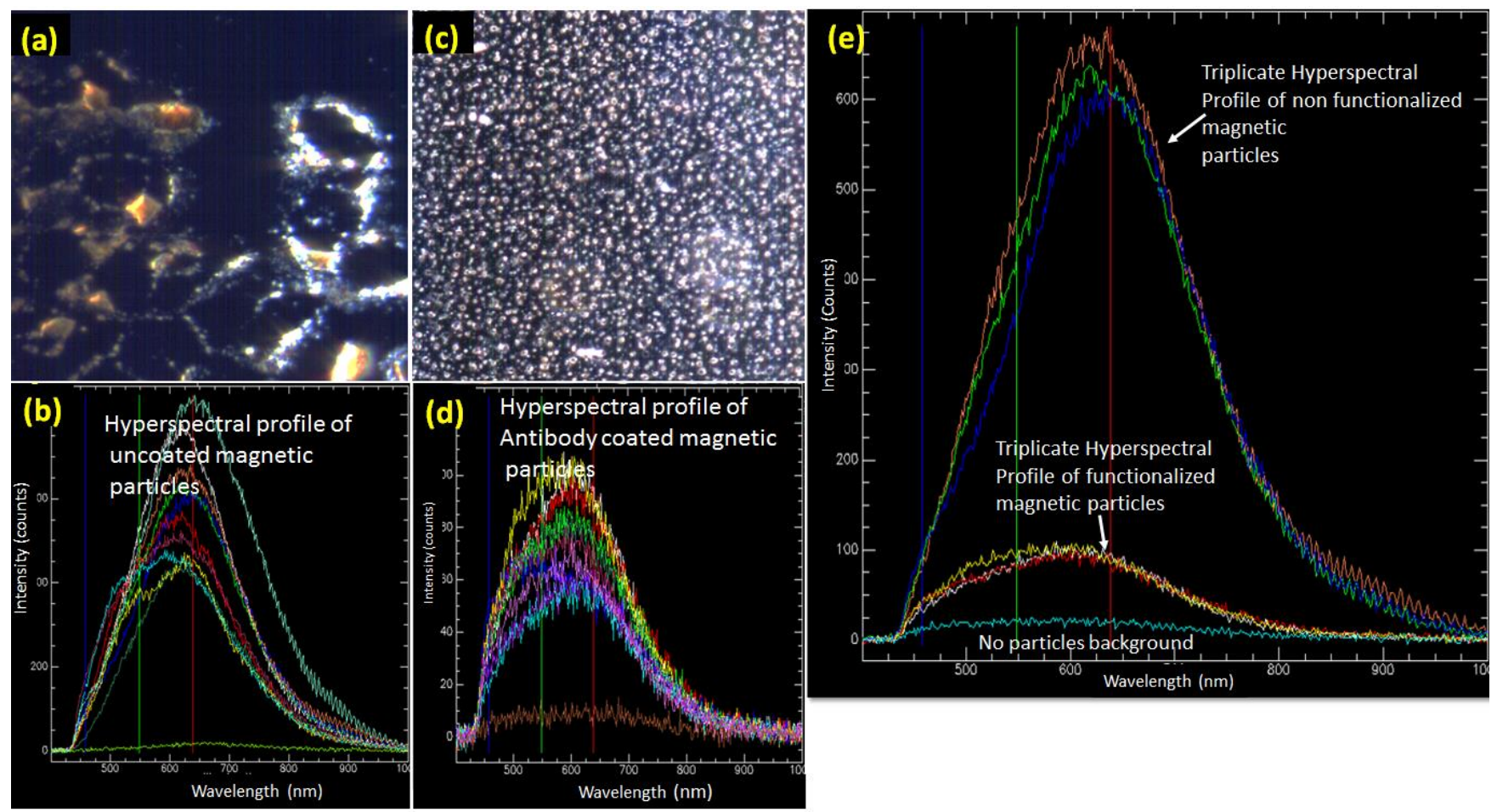

Figure 1. Hyperspectral analysis. (a) Non-coated MNPs, (b) Spectral profile of non-coated MNPs, (c) Antibody functionalized magnetic nanoparticles (AFMNPs), (d) Spectral profile of AFMNPs, and (e) overlay of the AFMNPs vs non MNPs profiles.

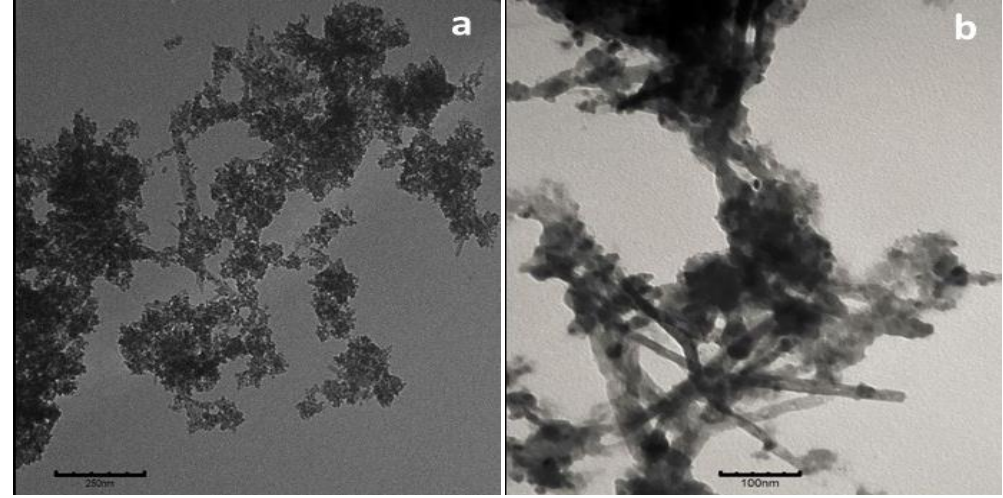

Figure 2. Transmission electron micrographs. (a) Nonfunctionalized MNP vs (b) AFNMPs

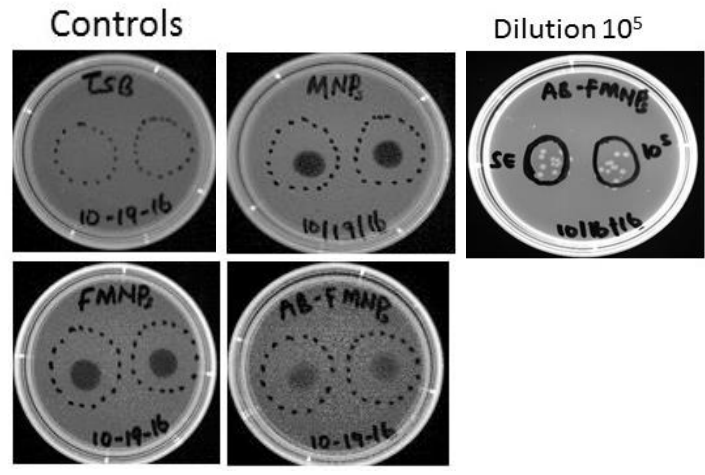

Figure 3. $S$. Enteritidis capture with AFMNPs

\section{References:}

1. Centers for Disease Control and Prevention. (2011, July 15).

2. S. Oliver, B. Jayarao, and R. Almeida (2005). Foodborne Pathog. Dis, 2:115-129.

3.Lopez, B., \& Merkoci, A. (2011).Trends Food Sci Technol , 2:625-39.

4. Inbaraj, B. S., \& Chen, B. (2016). J. Food. Drug. Anal., 24:15-28.

5. Valdes, et al. (2009). Microchim. Acta, 166:1-19.

6. Sonawane, et al. (2014). European J. Nutr. Food. Saf., 4:301-17.

7. Wang, et al (2014). Anal. Chem., 86:1671-1678.

8. Zhang, D., Carr, D. J., \& Alocilja, E. C. (2008). Biosens. Bioelectron., 24:1377-1381.

9. Supported by the USDA/NIFA/CBG 2012-38821-20056; US Department of Defense \# FA9550-14-10330 and \#W911NF-14-1-0139. 